OPEN ACCESS

Edited by:

Deepak Kumar Kaushik,

University of Calgary, Canada

Reviewed by:

Ines Moreno-Gonzalez,

University of Malaga, Spain

Valentina Echeverria Moran,

Bay Pines VA Healthcare System,

United States

*Correspondence:

Yajing $M i$

miyajing@163.com

Xingchun Gou

gouxingchun@189.cn

Received: 23 January 2020 Accepted: 01 May 2020

Published: 09 June 2020

Citation:

Han X, Zhang T, Liu H, Mi Y and Gou X (2020) Astrocyte Senescence and Alzheimer's Disease: A Review.

Front. Aging Neurosci. 12:148.

doi: 10.3389/fnagi.2020.00148

\section{Astrocyte Senescence and Alzheimer's Disease: A Review}

\author{
Xiaojuan Han, Tianying Zhang, Huanhuan Liu, Yajing Mi* and Xingchun Gou* \\ Shaanxi Key Laboratory of Brain Disorders \& Institute of Basic and Translational Medicine, Xi'an Medical University, \\ Xi'an, China
}

Astrocytes are the largest group of glial cells in the brain and participate in several essential functions of the central nervous system (CNS). Disruption of their normal physiological function can lead to metabolism disequilibrium and the pathology of CNS. As an important mechanism of aging, cellular senescence has been considered as a primary inducing factor of age-associated neurodegenerative disorders. Senescent astrocytes showed decreased normal physiological function and increased secretion of senescence-associated secretory phenotype (SASP) factors, which contribute to $A \beta$ accumulation, tau hyperphosphorylation, and the deposition of neurofibrillary tangles (NFTs) in Alzheimer's disease (AD). Astrocyte senescence also leads to a number of detrimental effects, including induced glutamate excitotoxicity, impaired synaptic plasticity, neural stem cell loss, and blood-brain barrier (BBB) dysfunction. In this review article, we have summarized the growing findings regarding astrocyte senescence and its putative role in the pathologic progress of AD. Additionally, we also focus on the significance of targeting astrocyte senescence as a novel and feasible therapeutic approach for AD.

Keywords: Alzheimer's disease, astrocytes, senescence, senescence-associated secretory phenotype, senolytic drugs, tau aggregate

\section{INTRODUCTION}

Alzheimer's disease $(\mathrm{AD})$ is a chronic degenerative disorder of the brain related to progressive decline of memory and cognition (McGeer and McGeer, 2007; Long and Holtzman, 2019). The disease is characterized by brain atrophy, extracellular accumulation of beta-amyloid peptide (A $\beta$ ) (Glenner and Wong, 1984; Hsiao et al., 1996), neurofibrillary tangles (NFTs) composed of hyperphosphorylated tau protein (Goedert et al., 2006), and loss of synapses and dysfunctions of neurotransmission (DeKosky and Scheff, 1990; Rajmohan and Reddy, 2017), as well as neuroinflammation (Heneka et al., 2015).

Many of the cellular pathologies of AD present on neurons, such as neuronal extracellular deposits of $A \beta$, intracellular deposition of neurofibrillary tangles (NFTs), and Lewy bodies (Hansen et al., 1990; Hardy and Higgins, 1992; Goedert et al., 2006). These classical pathologies are still central to diagnosing $\mathrm{AD}$. However, although neurons have significant correlations with $\mathrm{AD}$, other cell types and factors in the brain may also contribute to cognitive decline during AD. Additionally, astrocytes are the major glial cells and are vital for the normal physiological functions of the central nervous system (CNS) (Sofroniew and Vinters, 2010; Matias et al., 2019). They perform critical roles in regulation of homeostasis and metabolism of the neurons, mediating uptake and recycling 
of neurotransmitters. Astrocytes also play a key role in maintenance of the blood-brain barrier (BBB). They also act as modulators of synaptic plasticity and transmission, supporting the view that astrocytes play an integral role in the initiation and progression of cognitive decline and AD (Acosta et al., 2017; Palmer and Ousman, 2018; Munger et al., 2019).

Aging is considered the most significant risk factor for the occurrence and development of AD (Kritsilis et al., 2018). The incidence of $\mathrm{AD}$ has been shown to increase with advancing age and cellular senescence (Baker et al., 2011). Studies regarding to the link and role of senescence in age-related diseases have become increasingly common, and are gradually becoming a new research area (Bhat et al., 2012; Baker and Petersen, 2018). Transcriptome analysis of $\mathrm{AD}$ and the aged human brain showed neurons and other non-neuronal CNS cell types including astrocytes, microglia, and oligodendrocytes displayed senescence-associated phenotypes (Salminen et al., 2011; Bhat et al., 2012; Boccardi et al., 2015; Boisvert et al., 2018; Bussian et al., 2018; Zhang P. et al., 2019). Although studies regarding astrocyte senescence during $\mathrm{AD}$ and the effects of senescent astrocytes in $\mathrm{AD}$ progression have recently increased, an understanding of its precise mechanisms is still lacking. Here, we review our current view on the role of astrocyte senescence in the pathogenesis of $\mathrm{AD}$.

\section{ASTROCYTES}

Astrocytes are the most abundant type of glial cells in the brain and are classified into two main groups: fibrous astrocytes and protoplasmic astrocytes (Sofroniew and Vinters, 2010). Fibrous astrocytes are characterized by the presence of numerous fibrils in their cytoplasm and are distributed mainly in the white matter. Protoplasmic astrocytes are typically located in the gray matter and have thick, short, highly branched processes with fewer fibrils (Sofroniew and Vinters, 2010). Despite heterogeneity of astrocytic subtype, these glia are responsible for multifarious complex and essential functions for CNS physiology, including the provision of nutrients to the neuron, regulation of synaptic plasticity, releasing transmitters (called gliatransmitters) in a $\mathrm{Ca}^{2+}$-dependent manner, supporting the blood-brain barrier (BBB), and maintaining the extracellular ion balance (Hussaini and Jang, 2018; Verkhratsky and Nedergaard, 2018).

Astrocytes have been shown to become activated in response to various stimuli and diseases of the CNS. One common and classical feature of reactive astrocytes is the release of a variety of effector molecules including chemokines, cytokines, and proteases (Liddelow et al., 2017; Ahmad et al., 2019). Interestingly, these factors overlap with the secretions of senescent astrocytes (Boisvert et al., 2018). The proteins associated with astrocyte activation, glial fibrillary acidic protein (GFAP) and vimentin, increased during aging (Porchet et al., 2003). Also, senescent astrocytes share many of the similar phenotypes with A1-like reactive astrocytes, including cellular morphological change and proinflammation secretions. It is possible that many previous studies, which focused on reactive astrocytes, may have been focusing on senescent astrocytes.
Although Cohen et al. reviewed the different features between reactive and senescent astrocytes, it is necessary to elucidate the characteristics of astrocyte senescence further (Cohen and Torres, 2019). In the next section, we will describe the phenotypes of senescent astrocytes in more detail.

\section{ASTROCYTE SENESCENCE: ASTROSENESCENCE}

According to Cohen et al., astrocytes can initiate a senescence program similar to that of other cell types in response to various stressors, termed "astrosenescence" (Cohen and Torres, 2019). Ponten and Macintyre (1968) compared the characteristics of glial cells isolated from malignant and benign tissue to examine the effect of long-term culturing on CNS cells. This study indicated that primary cells from normal tissue had a limited lifetime and stopped dividing after a set number of passages. This might be the first study to examine replicative senescence in glia cells. With time, numerous studies demonstrated that after exhausted replication, oxidative stress, proteasome inhibition, high glucose, or HIV infection, astrocytes show changes in several classical hallmarks of cellular senescence.

Primary astrocytes isolated from the cerebral cortex that underwent replicative senescence displayed a series of established markers of cellular senescence including a growth arrest, increased expression of senescence-associated genes $p 53$ and $p 21^{W A F 1}$, and increased senescence-associated $\beta$-galactosidase (SA- $\beta$-Gal) activity (Evans et al., 2003; Blasko et al., 2004; Pertusa et al., 2007). Astrocytes have shown to undergo stressinduced premature senescence as well. For example, after ionizing radiation or $\mathrm{H}_{2} \mathrm{O}_{2}$ or proteasome inhibitor treatment, both human and mouse astrocytes displayed classical senescence features, such as decreased proliferation, increased SA- $\beta-\mathrm{Gal}$ activity, and the upexpression of $p 53, p 21^{W A F 1}$, and $p 16^{I N K 4 A}$ (Bitto et al., 2010; Turnquist et al., 2019). Notably, astrocytes were found to be more sensitive to senescence-inducing stimuli than fibroblasts (Gorg et al., 2015, 2018). Also, A $\beta$ oligomers can induce cellular senescence and promote production of senescence-associated secretory phenotypes (SASPs) in human astrocytes (Mombach et al., 2015). Furthermore, human astrocytes infected with HIV showed signs of DNA damage and premature senescence (Cohen et al., 2017). Therefore, astrocytes have been shown to undergo cellular senescence in vitro and in vivo due to various stimuli and factors.

\section{CHARACTERISTICS OF ASTROCYTE SENESCENCE}

Cellular senescence is a catchall for a set of states in which cells stop dividing and then exhibit a multitude of cellular and molecular changes. Evidence suggests that there is a significant variation in the senescent phenotype that is dependent on both cell type and triggering insults (Gorgoulis et al., 2019). Senescent astrocytes exhibit both classic characteristics as well as other cell types and also demonstrated particular phenotypes (Bhat et al., 
2012; Buhlman, 2017; Baker and Petersen, 2018). Key features of senescent astrocytes include the following: permanent cell cycle arrest, altered morphology, increased GFAP and vimentin, chromatin alterations and formation of senescence-associated heterochromatic foci (SAHFs), upexpression of high-mobility group B (HMGB) proteins, reduced expression of nuclear lamina protein laminB1, downregulation of neurotrophic growth factors, and upregulation of SASP factors as well as SA- $\beta$-Gal (Bitto et al., 2010; Boccardi et al., 2015; Chinta et al., 2015; Boisvert et al., 2018; Turnquist et al., 2019; Figure 1).

\section{Cell Arrest and GFAP}

Typically, senescent astrocytes exhibit permanent cell cycle arrest as well as other cell types, which is thought to be regulated by the $\mathrm{p} 53 / \mathrm{p} 21^{\mathrm{WAF} 1}$ and $\mathrm{p} 16^{\mathrm{INK} 4 \mathrm{~A}} / \mathrm{pRB}$ pathway (Evans et al., 2003; Bitto et al., 2010; Turnquist et al., 2019). p $21^{\text {WAF1 }}$ is namely the CIP/KIP (CDK interacting protein/kinase inhibitory protein) that is capable of inhibiting $\mathrm{CDK} 2$, but paradoxically, it is also necessary for cell cycle progression (Hernandez-Segura et al., 2018). In the context of astrocyte senescence, p53 upregulates the expression of $\mathrm{p} 21^{\mathrm{WAF} 1}$, which inhibits cyclin D-dependent kinase CDK2 activity and the initial cell cycle arrest. Interestingly, this p53-dependent stable proliferative arrest was independent of telomere erosion in human astrocyte in vitro study (Evans et al., 2003). p16 ${ }^{\mathrm{INK} 4 \mathrm{~A}}$ is a member of the INK4A family that mediates permanent cell cycle arrest by inhibiting CDK4 and CDK6, which leads to retinoblastoma protein (RB) hypophosphorylation, blocking cell cycle entry to the $S$ phase (Hernandez-Segura et al., 2018). Importantly, as its expression increased in the brain with time, $\mathrm{p} 16^{\mathrm{INK} 4 \mathrm{~A}}$ is also a biomarker of natural brain aging (Berchtold et al., 2008; Baker and Petersen, 2018).

Additionally, GFAP is a class III intermediate filament protein, which is the most widely used marker for astrocytes (Eun et al., 2016). In the human brain, the level of GFAP was significantly increased in the hippocampus in people over 65 years of age. GFAP upexpression has been the general change observed in astrocyte senescence in vitro and in vivo (Nichols et al., 1993; Boisvert et al., 2018; Lye et al., 2019). Larsson et al. have demonstrated that cell proliferation in the granular layer of the dentate gyrus is increased after knockout GFAP/vimentin

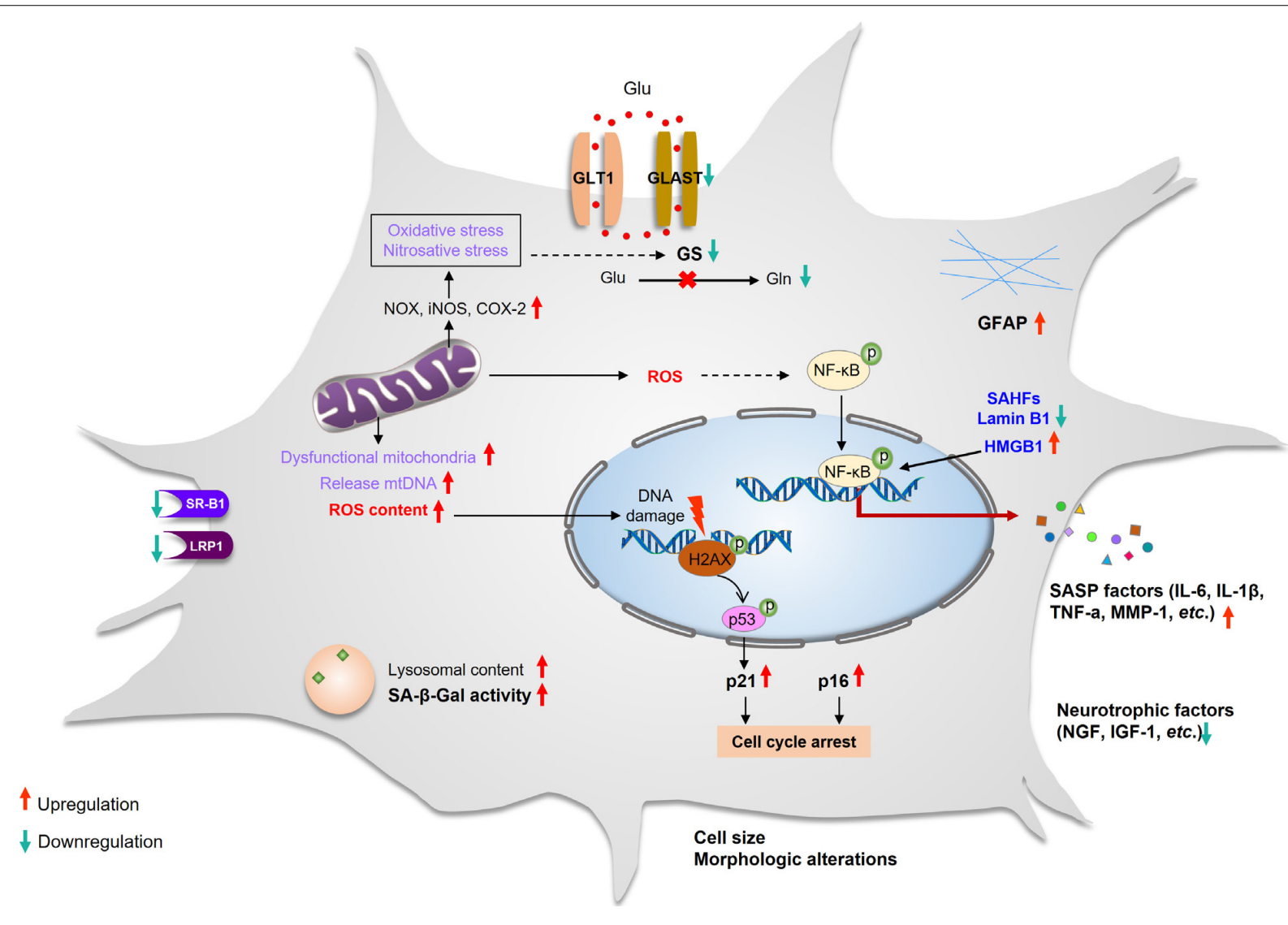

FIGURE 1 | Characteristics of astrocyte senescence. Senescent astrocytes that have undergone cell cycle arrest and have enlarged sharply. The expression of GFAP increased, whereas the expression of glutamate transporters (GLAST and GS) and SR-B1 and LRP1 decreased. The lysosomal content has increased, and the lysosomes have high $\beta$-galactosidase activity and dysfunctional mitochondria that produce high levels of ROS and release mtDNA. They have DNA damage and SAHFs, and their nuclear integrity is compromised due to the loss of laminB1. The elevated ROS activates the NF-kB pathway and promotes SASP production. The secretion of neurotrophic factors is decreased. GFAP, glial fibrillary acidic protein; GLAST, glutamate aspartate transporter; GLT-1, glutamate transporter-1; GS, glutamine synthetase; iNOS, inducible nitric oxide synthase; LRP1, lipoprotein receptor-related protein 1; NOX, NADPH oxidase; ROS, reactive oxygen species; SAHFs, senescence-associated heterochromatic foci; SASP, senescence-associated secretory phenotype; SR-B1, scavenger receptor B1. 
(Larsson et al., 2004). Several studies have demonstrated that the expression of GFAP appears to increase with aging in rodents and human astrocytes (Iram et al., 2016), although activated astrocytes also involve the upregulation expression of GFAP (Liddelow et al., 2017). This implies that astrocyte senescence associated with an increase in GFAP expression, which results in the upregulation of GFAP, may be a new biomarker of astrocyte senescence (Figure 1).

\section{Nuclear Changes}

In senescent cells, chromatin alterations and remodeling including formation of SAHFs and nuclear DNA enriched for histones modification were stained densely by DAPI (Kosar et al., 2011). These nuclear changes have been associated with attenuated expression of proliferation-promoting genes, which leads to irreversible cell cycle arrest of senescence (HernandezSegura et al., 2018; Gorgoulis et al., 2019). Unlike $\gamma \mathrm{H} 2 \mathrm{AX}$, which is a ubiquitously expressed DNA damage response (DDR) marker, the formation of SAHFs in senescent cells varies between cell types (Seoane et al., 2017; Gorgoulis et al., 2019). Studies have shown that cultured human astrocytes displayed both increased levels of $\gamma \mathrm{H} 2 \mathrm{AX}$ and formation of SAHFs (Myung et al., 2008; Bitto et al., 2010; Souza et al., 2015; Seoane et al., 2017; Figure 1). Senescent astrocytes showed nuclear enlargement, which is generally present in specific cell types such as fibroblasts and CNS cells (Yoon et al., 2016; Bang et al., 2019). When DNA damage and DNA repair occur during the cell cycle, the size of the cell and nucleus is slightly increased (Amodeo and Skotheim, 2016). It seems that the enlargement of the nucleus in senescent cells may be caused by DNA damage and cell cycle arrest.

Senescent astrocytes also display changes in nuclear morphology and integrity of the nuclear envelope owing to the downregulation of nuclear lamina proteins, such as lamin B1 (Freund et al., 2012). Additionally, HMGB1 plays a crucial role in DDR and cellular inflammation (Enokido et al., 2008; Castiglioni et al., 2011), which is increased in astrocytes with aging (Enokido et al., 2008; Figure 1).

\section{Senescence-Associated Secretory Phenotype}

Oxidative stress-induced and cultured senescent astrocytes cause several transcriptomic changes. More specifically, genes associated with proinflammation cytokines, such as interleukin (IL)-6, IL-8, chemokines, and proteinases were upregulated (Crowe et al., 2016; Hou et al., 2018). These proinflammation factors are termed the SASPs, which are considered to be a downstream consequence of the DDR (Salminen et al., 2011; Seoane et al., 2017). Additionally, $A \beta$ peptides and environmental toxins, such as ammonia and paraquat, have also been shown to induce senescence in cultured astrocytes with increased production of SASP. SASP is highly heterogeneous in senescent astrocytes that are induced by different stimuli (Bhat et al., 2012; Crowe et al., 2016; Clarke et al., 2018; Hou et al., 2018). However, the high expression of IL- 6 is a more common feature in astrocyte senescence (Blasko et al., 2004; Hou et al., 2017).

Several studies indicated that SASP-related genes upregulated in senescent astrocytes is mediated by the p38/MAPK and NF- $\kappa$ B pathway (Chien et al., 2011; Salminen et al., 2011; Mombach et al., 2015). Bhat et al. reported that inhibition of p38/MAPK activity both in presenescent and senescent human astrocytes suppress the increased level of SASP factors (Bhat et al., 2012). Following the hypothesis that DNA damage is an essential driver of SASP, HMGB1 may be another important regulator. In the brain, HMGB1 is upexpressed in astrocytes during aging (Enokido et al., 2008). Davalos et al. reported that HMGB1 could increase $\mathrm{NF}-\kappa \mathrm{B}$ transactivation efficiency through interaction with $\mathrm{NF}-\kappa \mathrm{B}$ complexes and therefore augment and strengthen the inflammatory reaction of the SASP (Qiu et al., 2010; Davalos et al., 2013). Recently, the cyclic GMP-AMP synthase (cGAS)/ stimulator of interferon (IFN) genes (STING) pathway, NOTCH signaling, and mammalian target of rapamycin (mTOR) signaling have also been shown to regulate SASP in fibroblast and other cell types (Herranz et al., 2015; Capell et al., 2016; Hoare et al., 2016; Loo et al., 2019). However, the mechanisms involved in CNS cells have not been extensively studied. SASPs have been shown to operate as autocrine and paracrine signals to reinforce the senescent state and induce senescence or degenerative changes of the surrounding bystander cells (Coppe et al., 2010; Acosta et al., 2013). These observations indicate that SASPs can generate a lowlevel, chronic inflammation and an age-dependent detrimental cycle to strengthen the senescence state and enhance age-related neurodegenerative disorders.

\section{Lysosomal and Mitochondrial Dysfunction}

The upregulation of lysosomal proteins and increased lysosomal content is the main characteristic of cellular senescence (Lee et al., 2006). Enhanced lysosomal content can be detected by measuring the activity of the lysosomal enzyme senescence-associated betagalactosidase (SA- $\beta$-Gal), which is measured at $\mathrm{pH} 6.0$ using in situ staining with the chromogenic substrate $\mathrm{X}$-gal (Kurz et al., 2000; Lee et al., 2006). Therefore, SA- $\beta$-Gal is used as the most common hallmark for detecting senescent cells. Senescent astrocytes showed elevated SA- $\beta$-Gal activity in vitro and in vivo (Evans et al., 2003; Bitto et al., 2010; Bhat et al., 2012; Cohen and Torres, 2019). Consistent with others, our previous research also demonstrated the accumulation of dysfunctional lysosomes in stress-induced premature senescence (SIPS) (Han et al., 2016; Tai et al., 2017).

It is known that the number of mitochondria increases during senescence. However, the membrane potential of mitochondria is decreased, leading to intensified ROS production and the release of mitochondrial DNA (mtDNA) (Passos et al., 2007). Meanwhile, elevated mtDNA in the cytoplasm can lead to STING-meditated SASP production (Li and Chen, 2018). Enhanced mitochondria content during senescence could be the result of the accumulation of dysfunctional mitochondria and reduced mitochondrial fission (Tai et al., 2017; Kim et al., 2018). In addition, impaired mitophagy is also likely to contribute to dysfunctional mitochondria accumulation in senescent cells (Fivenson et al., 2017; Vasileiou et al., 2019). Studies using either senescent astrocytes from aged rats or in vitro culturing showed that mitochondrial dysfunction and damage was associated with an increase in oxidative/nitrosative stress, RNA oxidation, 
upregulation of ROS, and inducible nitric oxide synthase (iNOS) expression levels (Figure 1; Pertusa et al., 2007; Bellaver et al., 2017; Bang et al., 2019).

\section{Glutamate Signaling Dysfunction}

Due to the high expression of glutamate transporters, GLAST (human homologs, EAAT1) and GLT-1 (human homologs, EAAT2) (Zhang X. et al., 2019), astrocytes can further regulate neuronal function via the efficient uptake of the synaptically released excitatory and inhibitory neurotransmitters glutamate and $\gamma$-aminobutyrate (GABA). Glutamate is the primary excitatory neurotransmitter in the mammalian CNS and is critical for learning and memory (Verkhratsky and Kirchhoff, 2007). Nevertheless, excessive extracellular glutamate levels lead to neuronal death as a result of glutamate excitotoxicity (Danbolt, 2001). The released glutamate from neurons is mainly taken up into astrocytes by GLAST and GLT- 1 and is then converted to glutamine by glutamine synthase (GS), which is expressed in astrocytes (Pajarillo et al., 2019; Zhang X. et al., 2019). The expression and activity of GS are age dependent. The protein level of GS has been found to decrease dramatically in aged astrocytes (Bellaver et al., 2017; Shi et al., 2017). In addition, the expression of the glutamate transporter GLAST displays an age-dependent decrease. However, there was an increase in the expression of GLT-1 in senescent astrocytes (Bellaver et al., 2017). Other research has shown only reduced expression and activity of GS, with no changes observed in the expression of GLAST andGLT-1 in senescent astrocytes (Boisvert et al., 2018). Additionally, the activity of GS is very sensitive to nitrosative and oxidative stress (Knorpp et al., 2006; Bellaver et al., 2017). Therefore, oxidative stress could decrease the ability of astrocytes to supply metabolic substrates to neurons and aggravate the impairment of GS activity (Crowe et al., 2016; Gonzalez-Reyes et al., 2017). It is likely that senescent astrocytes with reduced capacity for glutamate uptake and clearance contribute to glutamate excitotoxicity in neurodegenerative diseases.

\section{Cholesterol Synthesis}

Cholesterol is an essential metabolic substrate for the normal physiological functions of the brain, supporting neuronal homeostasis, synaptic integrity, and receptor function. In the $\mathrm{CNS}$, cholesterol content is largely independent of dietary intake because of the existence of the BBB (Giudetti et al., 2016). Astrocytes are thought to be crucial in brain cholesterol synthesis and transport owing primarily to their expression of sterol regulatory element-binding protein 2 (SREBP2) and apoE (Ferris et al., 2017). SREBP2 is an important transcription factor that regulates the expression of HMG-CoA reductase (HMGCR), the rate-limiting enzyme of cholesterol synthesis (Kim et al., 2010). In senescent astrocytes, the expression of HMGCR and genes associated with cholesterol synthesis is significantly decreased, while the mRNA level of cholesterol transport-related genes is increased (Boisvert et al., 2018). It appears that there is an overall dysregulation of cholesterol metabolism in senescent astrocytes, and this may lead to decreased synaptic support.

\section{FUNCTIONAL CONSEQUENCES OF SENESCENT ASTROCYTES IN ALZHEIMER'S DISEASE}

Alzheimer's disease $(\mathrm{AD})$ is a progressive neurodegenerative disease causing cognitive, memory, and behavioral dysfunctions in older age. The pathophysiological mechanism of AD has been extensively studied for many years, which has led to several hypotheses, such as the $\mathrm{A} \beta$, tau, cholinergic, and inflammation hypotheses (Hardy and Higgins, 1992; McGeer and McGeer, 2007; Hampel et al., 2018).

Numerous studies have shown that impaired astrocytes are involved in the initiation and progression of $\mathrm{AD}$ (Acosta et al., 2017; Gonzalez-Reyes et al., 2017; Matias et al., 2019). One most likely mechanism is astrocytic neuroinflammation (Benzing et al., 1999; Blasko et al., 2004). Also, a common feature of astrocyte senescence is the production of proinflammatory factors known as the SASP. In addition, studies have shown evidence that astrocytes surrounding $A \beta$ plaques are positive for IL-6, a key SASP component (Bhat et al., 2012; Munger et al., 2019). All these data support the view that astrocyte senescence is a key and novel contributor to $\mathrm{AD}$ pathogenesis.

The first study to investigate the role of astrocyte senescence in $\mathrm{AD}$ involved human astrocytes treated with the $\mathrm{A} \beta_{1-42}$ oligomer in vitro (Bhat et al., 2012). After treatment, astrocytes displayed classical phenotypes of cellular senescence including increased SA- $\beta$-Gal activity and increased $\mathrm{p} 16^{\mathrm{INK} 4 \mathrm{~A}}$ expression. Most notably, not only was there an age-dependent increase in p16-positive astrocytes in the frontal cortex tissue, there was a further increase in senescent astrocytes in brain tissue from $\mathrm{AD}$ patients compared to non-diseased tissues from age-matched individuals (Bhat et al., 2012; Turnquist et al., 2016). Also, a recent study suggested that removal of senescent astrocytes and microglia by senolytic agents or genetic ablation prevents or inhibits NFTs formation and neurodegeneration in $\mathrm{AD}$ and tauopathy mouse models (Bussian et al., 2018; Mendelsohn and Larrick, 2018), suggesting that astrocyte senescence contributes to the pathogenesis of neurodegeneration in $\mathrm{AD}$. In this part, we summarize the data that demonstrate the effects of senescent astrocytes in $\mathrm{AD}$ (Figure 2).

\section{$A \beta$ Accumulation}

Inefficient $\mathrm{A} \beta$ clearance is detrimental in $\mathrm{AD}$. Many studies have shown that astrocytes play an important role in $\mathrm{A} \beta$ clearance and degradation (Nicoll and Weller, 2003; Liu et al., 2017). Astrocyte receptors involved in uptake and clearance of $A \beta$ are the low-density lipoprotein receptor-related protein 1 (LRP1) and scavenger receptor B1 (SR-B1) (Basak et al., 2012; Ries and Sastre, 2016). However, the mechanisms governing the receptormediated uptake of $A \beta$ are not fully understood. Notably, in aged astrocytes, the expressions of LRP1 and SR-B1 are reduced (Iram et al., 2016), suggesting the ability of senescent astrocytes to uptake and degrade $A \beta$ may be impaired.

In $\mathrm{AD}$, senescent astrocytes are observed in regions surrounding $A \beta$ plaque and the appearance of SA- $\beta$-Gal-positive 


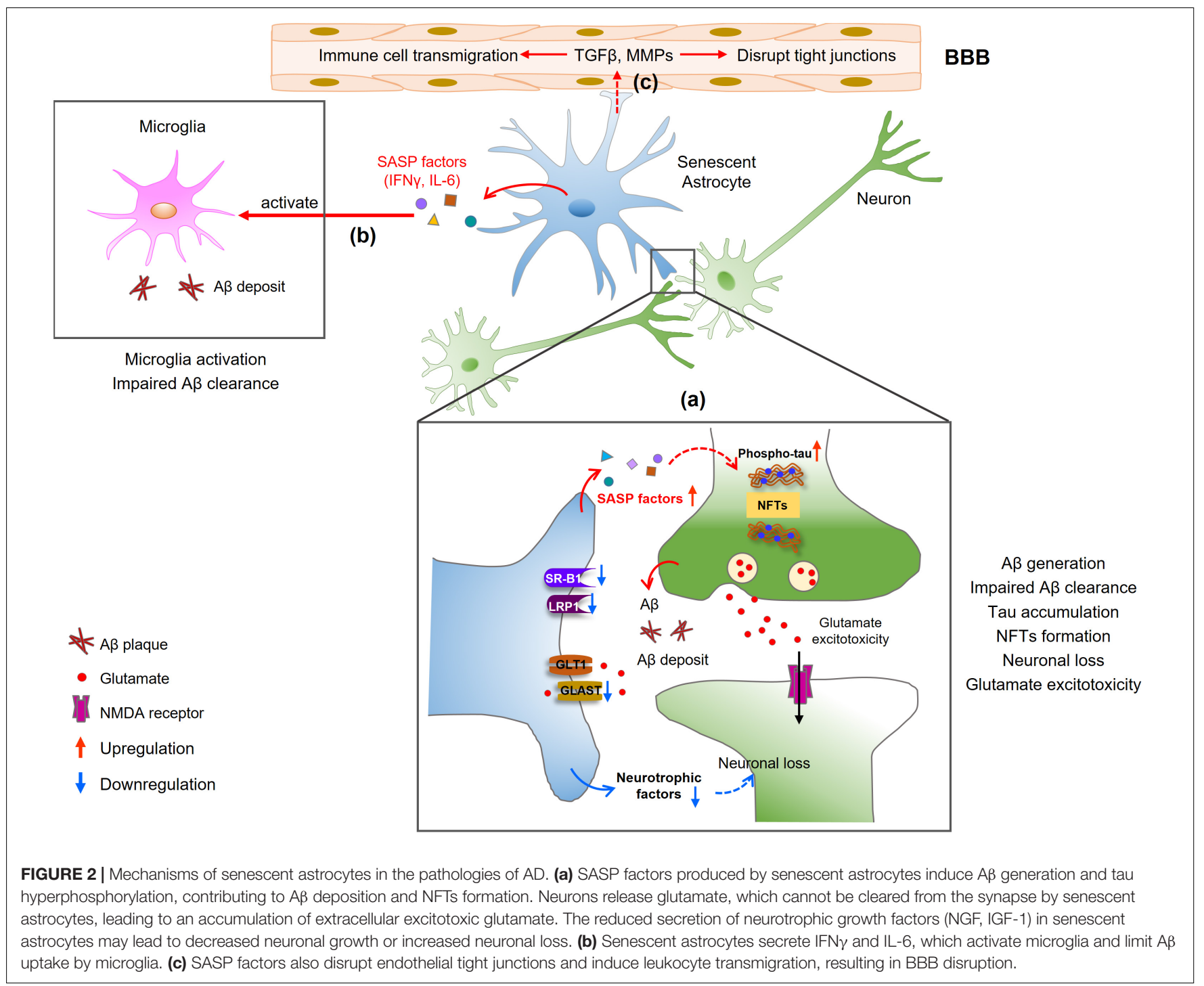

astrocytes was earlier than $A \beta$ accumulation (Bhat et al., 2012). The expression of BACE1 and $\gamma$-secretase subunits PS1, PS2, and PEN2 is increased by cellular stress in astrocytes (Grolla et al., 2013; Frost and Li, 2017). Subsequent studies have demonstrated that $A \beta_{1-42}$ itself can induce astrocyte senescence and further induce the astrocytic APP and $\beta$-secretase processing, resulting in a further increase in oligomeric and fibrillary $\mathrm{A} \beta$ (Frost and $\mathrm{Li}$, 2017; Garwood et al., 2017). Additionally, the proinflammatory factors secreted by senescent astrocytes can also increase APP expression and $A \beta$ generation in neurons (Del Bo et al., 1995). This means the higher release of SASP factors reduces the ability of the glia cells to promote $A \beta$ clearance and facilitates its accumulation in the brain.

\section{Tau Accumulation and NFT Formation}

Abnormal phosphorylation of the microtubule-associated protein tau and the subsequent accumulation of NFTs are the major pathological mechanisms of AD. Many studies have documented the vital role of senescent astrocytes in tau hyperphosphorylation and NFT formation (Bussian et al., 2018; Mendelsohn and Larrick, 2018). Musi et al. (2018) demonstrated that neurons carrying NFTs themselves become senescent, causing toxicity to nearby neurons. They performed a transcriptomic analysis of NFT-containing human neurons from the postmortem AD brain and revealed increased levels of the cellular senescence hallmark. The expression of senescenceassociated genes, such as $p 21^{W A F 1}$ and SASP proinflammatory genes, was significantly upregulated in the brain of the $\mathrm{AD}$ transgenic mouse model with NFTs. The authors treated with senolytics to remove the senescent cells, and found a reduction in total NFT density, neuronal loss, and ventricular enlargement. Although they link the expression of biomarkers of cellular senescence with the appearance of the NFTs in the AD mouse model, a causal relationship is not established, and the specific cell types involved cannot be identified. A recent study by Bussian et al. (2018) found the accumulation of $\mathrm{p} 16^{\mathrm{INK} 4 \mathrm{~A}}$-expression senescent astrocytes and microglia in the MAPT ${ }^{\mathrm{P} 3015}$ PS19 mouse model of tau-dependent neurodegenerative disease. They then 
created a mouse strain (PS19/ATTAC) by crossing INK-ATTAC transgenic mice with the PS19 strain to remove p16-positive senescent cells through the administration of an inducer agent. They found that the clearance and removal of senescent astrocytes and microglial cells almost completely prevented hyperphosphorylated tau protein and NFT deposition in PS19/ATTAC mice, preserving cognitive function. Collectively, these results demonstrate that senescent astrocytes have a crucial role in tau accumulation and thus the etiopathology of tauassociated disease (Figure 2). These data also suggest a strong correlation between astrocyte senescence, $A \beta$ formation, and tau accumulation, but the underlying mechanism tying together these characteristics with respect to senescent astrocytes in $\mathrm{AD}$ remains unclear and inconclusive.

\section{Synaptic Dysfunction and Neuronal Loss}

Cognitive and memory impairment in AD is most likely caused by synapse loss and synapse dysfunction rather than mere neuronal loss or neuronal death (Terry et al., 1991; Hong et al., 2016). As the major glial cell types of the brain, astrocytes play a critical role in supporting neuronal growth and modulating synaptic function and transmission, yet the neuroprotective capacity of astrocytes decreased during aging (Pertusa et al., 2007; Yu et al., 2017). Although the mechanism of synapse dysfunction has not yet been fully elucidated, it is most likely a combination of $A \beta$ plaque deposition, tau accumulation, and lesions (Miller et al., 2014; Piacentini et al., 2017). Astrocytes exhibit a senescencelike phenotype around $A \beta$ plaques and NFTs in the brains of $\mathrm{AD}$ patients and $\mathrm{AD}$ mouse models (Bhat et al., 2012; Lok et al., 2013; Musi et al., 2018), and synapse dysfunction is also found mainly surrounding dense-core $A \beta$ plaques (Koffie et al., 2009). These studies provide support for a relationship between senescent astrocytes and synaptic dysfunction or synapse loss in $\mathrm{AD}$ progression. In vitro, hippocampal neurons cocultured with senescent astrocytes showed suppressed synaptic maturation and transmission accompanied by a reduction in the size of synaptic vesicles (Kawano et al., 2012; Turnquist et al., 2016). The neuronal loss observed in $\mathrm{AD}$ can be attributed to the release of SASP factors such as IL-6. Furthermore, primary astrocytes from aged $5 \mathrm{xFAD}$ mice showed elevated SASP factor expression and neurotoxicity as well as impairment in supporting neuronal homeostasis (Iram et al., 2016). Moreover, senescent astrocytes secrete less neurotrophins such as nerve growth factor (NGF), insulin-like growth factor 1 (IGF-1), fibroblast growth factor 2 (FGF2), brain-derived neurotrophic factor (BDNF), and vascular endothelial growth factor (VEGF) (Bernal and Peterson, 2011; Turnquist et al., 2016; Bellaver et al., 2017), which may be lead to decreased neuronal growth and increased neuronal death in age-related neurological disorders.

Astrocytes also modulate synaptic properties via the abnormal release of the gliotransmitter GABA (Morel et al., 2017; PerezNievas and Serrano-Pozo, 2018). Moreover, senescent cortical astrocytes contribute to the impairment of synaptic plasticity and cognitive decline via a decline in the production of ATP, which is an important metabolic factor of neuronal activity (Lalo et al., 2014).

\section{Blood-Brain Barrier Dysfunction}

The BBB limits the migration of cells and diffusion of molecules freely entering and exiting from the brain. The BBB is crucial for maintaining homeostasis of the brain microenvironment, which consists of perivascular microglia, endothelial cells, pericytes, neurons, and astrocytic end feet (Yamazaki and Kanekiyo, 2017). The impairment function of any of these cells could result in disturbance to the BBB.

In fact, the $\mathrm{BBB}$ is seen to leak both in normal aging and AD (Montagne et al., 2015; Yamazaki and Kanekiyo, 2017). However, the underlying mechanism of how aging disrupts the integrity of the BBB remains inconclusive. Astrocytes, although not involved in the formation of the BBB, participate in its maintenance and regulation. Senescent astrocytes produce a variety of SASP factors that influence the permeability of the BBB (Spampinato et al., 2017; Boisvert et al., 2018; Clarke et al., 2018). For instance, matrix metalloproteinases (MMPs), nitric oxide (NO), and VEGFs cause endothelial apoptosis and disrupt endothelial tight junctions (TJs) by downregulation of TJ-related proteins, resulting in BBB disruption (Abbott, 2002; Horng et al., 2017; Spampinato et al., 2017). Some of these molecules include transforming growth factor- $\beta$ (TGF $\beta$ ), glial cell-derived neurotrophic factor (GDNF), basic fibroblast growth factor (bFGF), IL-6 and upregulate endothelial cell adhesion molecules (CAMs), which induce leukocyte transmigration (Rochfort and Cummins, 2015). Interestingly, recent research has shown that the accumulation of senescent vascular cells results in compromised BBB integrity and reduced microvessel TJ coverage (Yamazaki et al., 2016). These senescent vascular smooth muscle cells (VSMCs) also contribute to the brain inflammation environment through the upregulation of proinflammatory cytokine IL- 6 and chemokines, suggesting that senescent VSMCs have a crucial role in inducing age-dependent BBB breakdown. Yet, it is not clear whether the senescent astrocytes have a positive effect on VSMC senescence, and further studies should continue to explore the role of astrocyte senescence in increased $\mathrm{BBB}$ permeability during $\mathrm{AD}$.

\section{Activation of Microglia and Promotion of Chronic Inflammation}

Specifically, senescent astrocytes secrete SASP meditators such as IFN $\gamma$, CXCL10, IL-6, and TGF $\beta$, which are capable of inducing inflammation (Bhat et al., 2012; Stamouli and Politis, 2016). For example, IFN $\gamma$ is a potent regulatory cytokine that activates microglia and promotes inflammation in the $\mathrm{AD}$ brain (Blasko et al., 2004; Taylor et al., 2018). IL-6 is another typical SASP factor whose expression is upregulated in the aged brain and in those with $\mathrm{AD}$. Its overexpression has been shown to drive neurodegeneration in vitro (Bhat et al., 2012). Several SASP factors, including IL-6, IL-1 $\beta$, TNF- $\alpha$, MMP-1, MMP-3, and MMP-10, have also been found to be elevated in the cerebrospinal fluid (CSF) and serum of AD patients (Wood et al., 1993; BlumDegena et al., 1995; Leake et al., 2000; Horstmann et al., 2010; Gezen-Ak et al., 2013). This suggests that senescent astrocytes may sustain a proinflammatory status via the production of SASP factors and SASP-mediated microglia activation and 
inflammation may contribute to the pathogenesis of $\mathrm{AD}$ and aggravate the course of the disease.

\section{ASTROCYTE SENESCENCE AND OTHER NEURODEGENERATIVE DISEASES}

Senescent astrocytes are also more prominent in brain tissues from patients with other neurodegenerative diseases, such as Parkinson's disease (PD) (Chinta et al., 2018; Scott and Williams-Gray, 2018) and amyotrophic lateral sclerosis (ALS) (Turnquist et al., 2016; Vazquez-Villasenor et al., 2019). PD is the second most common age-related neurodegenerative disease, characterized by loss of neurons in the substantia nigra pars compacta (SNpc), accumulation of $\alpha$-synuclein, and presence of intraneuronal proteinaceous cytoplasmic inclusions (Lewy bodies) (Dauer and Przedborski, 2003). Increased levels of SASP-related factors, IL-1 $\beta$, IL-6, and TNF- $\alpha$, have been reported in the CSF, serum, and striatal dopaminergic regions of the patients with PD compared to controls (Mogi et al., 1994; Blum-Degena et al., 1995). Chinta et al. (2018) found that lamin B1 levels are decreased, while the expression of senescence-associated genes, $p 16^{I N K 4 A}$ and $p 21^{W A F 1}$, is increased in astrocytes of $\mathrm{SNpc}$ tissues in PD patients. Additionally, conditioned media from senescent astrocytes significantly reduced the viability of dopaminergic neurons and the proliferation and migration of neural progenitor cells (NPCs). Most importantly, they found that selective elimination of senescent astrocytes could repress the development of paraquatinduced neurodegenerative phenotypes associated with sporadic PD (Chinta et al., 2018), demonstrating that astrocyte senescence might be a critical mechanism for PD neurodegeneration. ALS is a fatal neurodegenerative disease characterized by the loss of upper and lower motor neurons (MNs). Postmortem brain tissue from ALS patients exhibits increased numbers of senescent astrocytes (Turnquist et al., 2016). This is further supported by animal models of ALS that show increased expression of the senescence marker $\mathrm{p} 16^{\mathrm{INK} 4 \mathrm{~A}}$ in GFAP-positive astrocytes within lumbar spinal cord sections that typically surround damaged motor neurons (Trias et al., 2019). Moreover, SASP cytokine IL-6 as well as p16 ${ }^{\mathrm{INK} 4 \mathrm{~A}}$ and $p 21^{\text {WAF1 }}$ were more remarkably upregulated in ALS compared to AD (Turnquist et al., 2016). Aged astrocytes are less supportive to motor neuron function (Das and Svendsen, 2015; Turnquist et al., 2016). Senescent astrocytes might exert their neurotoxic effect on neurons through the release of SASP factors, exacerbating neuroinflammation. However, it is not clear whether prevention or clearance of senescent cells in ALS could delay disease progression as is seen in PD models.

\section{THERAPEUTIC APPLICATIONS}

Currently, most of the therapeutic approaches of $\mathrm{AD}$ focus mainly on the modulation of $A \beta$ production by inhibiting $A \beta$ generation and enhancing $A \beta$ degradation or reducing tau protein deposits and NFT accumulation (Citron, 2010).
Others attempt at targeting apoE, neuroinflammation, metabolic dysfunction, and epigenetic changes (Long and Holtzman, 2019). Despite the fact that there are more than 100 different compounds in various stages of clinical trials being tested for use in early-, mid- or late-stage $\mathrm{AD}$, there are few efficacious therapeutic options available (Hara et al., 2019; Long and Holtzman, 2019). The failure of these trials emphasizes a great need for different pharmaceutical therapies to prevent or delay the progression of $\mathrm{AD}$.

Aging is a critical risk factor for most age-related neurodegenerative disease, including AD (Baker and Petersen, 2018; Kritsilis et al., 2018; Hara et al., 2019). A hallmark of aging is senescent cell accumulation. In recent years, a significant finding in aging and age-related diseases research is that selective elimination of senescent cells can extend lifespan and slow the progression of diseases in vivo without triggering negative side effects (Baker et al., 2011, 2016; Jeon et al., 2017). This strategy is referred to as senolysis. As mentioned earlier, a great number of studies indicated that astrocyte senescence plays a crucial role in the pathogenesis of AD. Senolytic therapeutic strategies that safely and effectively reduce the detrimental effects of senescent astrocytes, such as the neutralization of SASP or targeted clearance of senescent astrocytes, are gaining considerable attention in $\mathrm{AD}$.

\section{Alleviate Astrocyte Senescence and Decrease SASP Level}

Several lines of research suggest that astrocyte senescence occurs in the early stage of disease progression and may induce or aggravate other neurodegenerative pathologies. Thus, therapies to alleviate astrocyte senescence could prevent the onset of $\mathrm{AD}$ or delay its progress. In $\mathrm{AD}$, senescent astrocytes exert deleterious effects on neurons via both reduced secretion of neurotrophic growth factors (NGF, IGF-1) and elevated production of SASP factors (Turnquist et al., 2016). Turnquist et al. $(2016,2019)$ demonstrated that overexpression of $\Delta 133$ p53 or downregulation of $p 53 \beta$ can alleviate astrocyte senescence, repress SASP production, and consequently prevent neuronal apoptosis and loss. Additionally, in astrocytes, $\Delta 133 \mathrm{p} 53$ mediated upregulation of neurotrophic factors induced a neuroprotection effect. This shift secretion profile demonstrated that SASP may be a promising therapeutic method for slowing the progression of AD. There are two more commonly used SASP neutralization therapies: inhibiting SASP-initiated signaling and blocking the activity of particular components of the SASP.

As mentioned earlier, astrocytic SASP genes are upregulated in a $\mathrm{p} 38 / \mathrm{MAPK}$ and NF- $\mathrm{B}$ signaling-dependent manner. Thus, targeting the NF- $\mathrm{B}$ pathway decreased the production of SASP. Similarly, pharmacological inhibition of p38/MAPK abolished SASP secretion by senescent astrocytes. Yet, not all SASP factors are altered in senescent astrocytes. Because several SASP factors are essential to maintaining cell senescence, inhibition of proSASP signaling can increase the risk of cancer development (Coppe et al., 2010; Hoare et al., 2016). Alternatively, blockade of specific SASP factors, such as IL-6, is also a viable strategy. Specifically, using IL-6 monoclonal neutralizing antibodies, 
which are already approved for clinical applications, i.e., siltuximab (anti-human IL-6) or tocilizumab (anti-human IL$6 \mathrm{R})$, could show some promise.

\section{Clearance of Senescent Astrocytes (Senotherapy)}

In many age-related disorders such as osteoarthritis, atherosclerosis, and diabetes mellitus type 2, the removal of senescent cells of transgenic mice models has shown an impaired associated pathology and extended the healthy lifespan (Baker et al., 2011, 2016; Jeon et al., 2017). Success has also been observed in a mouse model of tau-associated pathogenesis. This study was the first to demonstrate a causal relationship between glial senescence and neurodegeneration (Bussian et al., 2018). In this study, Bussian et al. found accumulations of senescent astrocytes and microglia in tau-associated neurodegenerative disease model mice. Elimination of these senescent cells via a genetic approach can reduce tau deposition and prevent the degeneration of cortical and hippocampal neurons. Besides, a new set of pharmacological drugs has been proven to show a similar effect, termed senolytics, which include ABT263, quercetin, and dasatinib (Zhu et al., 2014). Indeed, these pharmacological agents have already been successful in preclinical and clinical trials in several age-associated diseases (reviewed by Childs et al., 2017). Most recently, Zhang X. et al. (2019) showed that clearance of senescent oligodendrocyte progenitor cells in $\mathrm{AD}$ model mice with senolytic agents could lessen the $A \beta$ plaque load, reduce neuroinflammation, and ameliorate cognitive deficits. This seno-therapeutic approach is currently being tested in neurodegenerative diseases and despite expected challenges and difficulties, more detailed investigation is warranted.

\section{CONCLUSION AND OUTLOOK}

Astrocytes undergo degeneration and senescence in the early stages of $\mathrm{AD}$ progression, which may alter the microenvironment

\section{REFERENCES}

Abbott, N. J. (2002). Astrocyte-endothelial interactions and blood-brain barrier permeability. J. Anat. 200, 629-638. doi: 10.1046/j.1469-7580.2002. 00064.x

Acosta, C., Anderson, H. D., and Anderson, C. M. (2017). Astrocyte dysfunction in Alzheimer disease. J. Neurosci. Res. 95, 2430-2447. doi: 10.1002/jnr.24075

Acosta, J. C., Banito, A., Wuestefeld, T., Georgilis, A., Janich, P., Morton, J. P., et al. (2013). A complex secretory program orchestrated by the inflammasome controls paracrine senescence. Nat. Cell Biol. 15, 978-990. doi: 10.1038/ ncb2784

Ahmad, M. H., Fatima, M., and Mondal, A. C. (2019). Influence of microglia and astrocyte activation in the neuroinflammatory pathogenesis of Alzheimer's disease: rational insights for the therapeutic approaches. J. Clin. Neurosci. 59, 6-11. doi: 10.1016/j.jocn.2018.10.034

Amodeo, A. A., and Skotheim, J. M. (2016). Cell-Size Control. Cold Spring Harb. Perspect. Biol. 8:a019083. doi: 10.1101/cshperspect.a019083

Baker, D. J., Childs, B. G., Durik, M., Wijers, M. E., Sieben, C. J., Zhong, J., et al. (2016). Naturally occurring p16(Ink4a)-positive cells shorten healthy lifespan. Nature 530, 184-189. doi: 10.1038/nature 16932 of the brain and contribute to early cognitive deficits. However, how senescent astrocytes actively and exactly contribute to the progression of $\mathrm{AD}$ is still to be fully characterized. Fortunately, targeting astrocyte senescence using a senolytics approach, among others, is beginning to emerge in $\mathrm{AD}$ treatments, with evidence in $\mathrm{AD}$ animal models already showing promise. Additionally, other cell types in the brain, including microglia (Flanary, 2005), oligodendrocytes (Zhang P. et al., 2019), neural stem cells (He et al., 2013), and neurons (Jurk et al., 2012), showed senescent phenotypes that are also involved in development of $\mathrm{AD}$. Therefore, the link between cellular senescence of other CNS cell types and $\mathrm{AD}$ needs to be further explored.

Furthermore, extensive astrocyte senescence has also been found in other age-related neurodegenerative diseases such as PD and ALS. This indicates that astrocyte senescence seems to be a common characteristic of neurodegeneration, and future research needs to examine this phenomenon in more detail. Additionally, the pathologies of neurodegenerative diseases induced by senescent astrocytes need to be further understood. Such future studies could increase our knowledge of cellular senescence and neurodegeneration medicine.

\section{AUTHOR CONTRIBUTIONS}

$\mathrm{XH}$ conceived and wrote the manuscript. TZ and HL contributed to manuscript preparation. YM and XG designed the concept of this study and discussed the results with all authors.

\section{FUNDING}

This work was supported by the National Natural Science Foundation of China (Grant Nos. 81971330, 81873740, 31900807) and the Natural Science Basic Research Program of Shaanxi (Program No. 2020JQ-878). We also thank the Youth Innovation Team of Shaanxi Universities for their support.

Baker, D. J., and Petersen, R. C. (2018). Cellular senescence in brain aging and neurodegenerative diseases: evidence and perspectives. J. Clin. Invest. 128, 1208-1216. doi: 10.1172/JCI95145

Baker, D. J., Wijshake, T., Tchkonia, T., LeBrasseur, N. K., Childs, B. G., van de Sluis, B., et al. (2011). Clearance of p16Ink4a-positive senescent cells delays ageing-associated disorders. Nature 479, 232-236. doi: 10.1038/nature10600

Bang, M., Kim, D. G., Gonzales, E. L., Kwon, K. J., and Shin, C. Y. (2019). Etoposide induces mitochondrial dysfunction and cellular senescence in primary cultured rat astrocytes. Biomol. Ther. 27, 530-539. doi: 10.4062/biomolther. 2019.151

Basak, J. M., Verghese, P. B., Yoon, H., Kim, J., and Holtzman, D. M. (2012). Low-density lipoprotein receptor represents an apolipoprotein E-independent pathway of Abeta uptake and degradation by astrocytes. J. Biol. Chem. 287, 13959-13971. doi: 10.1074/jbc.M111.288746

Bellaver, B., Souza, D. G., Souza, D. O., and Quincozes-Santos, A. (2017). Hippocampal astrocyte cultures from adult and aged rats reproduce changes in glial functionality observed in the aging brain. Mol. Neurobiol. 54, 2969-2985. doi: 10.1007/s12035-016-9880-8

Benzing, W. C., Wujek, J. R., Ward, E. K., Shaffer, D., Ashe, K. H., Younkin, S. G., et al. (1999). Evidence for glial-mediated inflammation in aged APPSW 
transgenic mice. Neurobiol. Aging 20, 581-589. doi: 10.1016/s0197-4580(99) 00065-2

Berchtold, N. C., Cribbs, D. H., Coleman, P. D., Rogers, J., Head, E., Kim, R., et al. (2008). Gene expression changes in the course of normal brain aging are sexually dimorphic. Proc. Natl. Acad. Sci. U.S.A. 105, 15605-15610. doi: 10.1073/pnas.0806883105

Bernal, G. M., and Peterson, D. A. (2011). Phenotypic and gene expression modification with normal brain aging in GFAP-positive astrocytes and neural stem cells. Aging Cell 10, 466-482. doi: 10.1111/j.1474-9726.2011.00694.x

Bhat, R., Crowe, E. P., Bitto, A., Moh, M., Katsetos, C. D., Garcia, F. U., et al. (2012). Astrocyte senescence as a component of Alzheimer's disease. PLoS One 7:e45069. doi: 10.1371/journal.pone.0045069

Bitto, A., Sell, C., Crowe, E., Lorenzini, A., Malaguti, M., Hrelia, S., et al. (2010). Stress-induced senescence in human and rodent astrocytes. Exp. Cell Res. 316, 2961-2968. doi: 10.1016/j.yexcr.2010.06.021

Blasko, I., Stampfer-Kountchev, M., Robatscher, P., Veerhuis, R., Eikelenboom, P., and Grubeck-Loebenstein, B. (2004). How chronic inflammation can affect the brain and support the development of Alzheimer's disease in old age: the role of microglia and astrocytes. Aging Cell 3, 169-176. doi: 10.1111/j.1474-9728.2004. 00101.x

Blum-Degena, D., Müller, T., Kuhn, W., Gerlach, M., Przuntek, H., and Riederer, P. (1995). Interleukin-1 $\beta$ and interleukin-6 are elevated in the cerebrospinal fluid of Alzheimer's and de novo Parkinson's disease patients. Neurosci. Lett. 202, 17-20. doi: 10.1016/0304-3940(95)12192-7

Boccardi, V., Pelini, L., Ercolani, S., Ruggiero, C., and Mecocci, P. (2015). From cellular senescence to Alzheimer's disease: the role of telomere shortening. Ageing Res. Rev. 22, 1-8. doi: 10.1016/j.arr.2015.04.003

Boisvert, M. M., Erikson, G. A., Shokhirev, M. N., and Allen, N. J. (2018). The aging astrocyte transcriptome from multiple regions of the mouse brain. Cell Rep. 22, 269-285. doi: 10.1016/j.celrep.2017.12.039

Buhlman, L. M. (2017). Parkin loss-of-function pathology: Premature neuronal senescence induced by high levels of reactive oxygen species? Mech. Ageing Dev. 161, 112-120. doi: 10.1016/j.mad.2016.06.008

Bussian, T. J., Aziz, A., Meyer, C. F., Swenson, B. L., van Deursen, J. M., and Baker, D. J. (2018). Clearance of senescent glial cells prevents tau-dependent pathology and cognitive decline. Nature 562, 578-582. doi: 10.1038/s41586-018-0543-y

Capell, B. C., Drake, A. M., Zhu, J., Shah, P. P., Dou, Z., Dorsey, J., et al. (2016). MLL1 is essential for the senescence-associated secretory phenotype. Genes Dev. 30, 321-336. doi: 10.1101/gad.271882.115

Castiglioni, A., Canti, V., Rovere-Querini, P., and Manfredi, A. A. (2011). Highmobility group box 1 (HMGB1) as a master regulator of innate immunity. Cell Tissue Res. 343, 189-199. doi: 10.1007/s00441-010-1033-1

Chien, Y., Scuoppo, C., Wang, X., Fang, X., Balgley, B., Bolden, J. E., et al. (2011). Control of the senescence-associated secretory phenotype by NFkappaB promotes senescence and enhances chemosensitivity. Genes Dev. 25, 2125-2136. doi: 10.1101/gad.17276711

Childs, B. G., Gluscevic, M., Baker, D. J., Laberge, R. M., Marquess, D., Dananberg, J., et al. (2017). Senescent cells: an emerging target for diseases of ageing. Nat. Rev. Drug Discov. 16, 718-735. doi: 10.1038/nrd.2017.116

Chinta, S. J., Woods, G., Demaria, M., Rane, A., Zou, Y., McQuade, A., et al. (2018). Cellular senescence is induced by the environmental neurotoxin paraquat and contributes to neuropathology linked to Parkinson's Disease. Cell Rep. 22, 930-940. doi: 10.1016/j.celrep.2017.12.092

Chinta, S. J., Woods, G., Rane, A., Demaria, M., Campisi, J., and Andersen, J. K. (2015). Cellular senescence and the aging brain. Exp. Gerontol. 68, 3-7.

Citron, M. (2010). Alzheimer's disease: strategies for disease modification. Nat. Rev. Drug Discov. 9, 387-398. doi: 10.1038/nrd2896

Clarke, L. E., Liddelow, S. A., Chakraborty, C., Munch, A. E., Heiman, M., and Barres, B. A. (2018). Normal aging induces A1-like astrocyte reactivity. Proc. Natl. Acad. Sci. U.S.A. 115, E1896-E1905. doi: 10.1073/pnas.1800165115

Cohen, J., D’Agostino, L., Wilson, J., Tuzer, F., and Torres, C. (2017). Astrocyte senescence and metabolic changes in response to HIV antiretroviral therapy drugs. Front. Aging Neurosci. 9:281. doi: 10.3389/fnagi.2017.00281

Cohen, J., and Torres, C. (2019). Astrocyte senescence: evidence and significance. Aging Cell 18:e12937. doi: 10.1111/acel.12937

Coppe, J. P., Desprez, P. Y., Krtolica, A., and Campisi, J. (2010). The senescenceassociated secretory phenotype: the dark side of tumor suppression. Annu. Rev. Pathol., 5, 99-118. doi: 10.1146/annurev-pathol-121808-102144
Crowe, E. P., Tuzer, F., Gregory, B. D., Donahue, G., Gosai, S. J., Cohen, J., et al. (2016). Changes in the transcriptome of human astrocytes accompanying oxidative stress-induced senescence. Front. Aging Neurosci. 8:208. doi: 10.3389/ fnagi.2016.00208

Danbolt, N. C. (2001). Glutamate uptake. Prog. Neurobiol. 65, 1-105.

Das, M. M., and Svendsen, C. N. (2015). Astrocytes show reduced support of motor neurons with aging that is accelerated in a rodent model of ALS. Neurobiol. Aging 36, 1130-1139. doi: 10.1016/j.neurobiolaging.2014.09.020

Dauer, W., and Przedborski, S. (2003). Parkinson's Disease. Neuron 39, 889-909.

Davalos, A. R., Kawahara, M., Malhotra, G. K., Schaum, N., Huang, J., Ved, U., et al. (2013). p53-dependent release of Alarmin HMGB1 is a central mediator of senescent phenotypes. J. Cell Biol. 201, 613-629. doi: 10.1083/jcb.201206006

DeKosky, S. T., and Scheff, S. W. (1990). Synapse loss in frontal cortex biopsies in Alzheimer's disease: correlation with cognitive severity. Ann. Neurol. 27, 457-464. doi: 10.1002/ana.410270502

Del Bo, R., Angeretti, N., Lucca, E., De Simoni, M. G., and Forloni, G. (1995). Reciprocal control of inflammatory cytokines, IL- 1 and IL-6, and $\beta$-amyloid production in cultures. Neurosci. Lett. 188, 70-74. doi: 10.1016/0304-3940(95) 11384-9

Enokido, Y., Yoshitake, A., Ito, H., and Okazawa, H. (2008). Age-dependent change of HMGB1 and DNA double-strand break accumulation in mouse brain. Biochem. Biophys. Res. Commun. 376, 128-133. doi: 10.1016/j.bbrc.2008.08.108

Eun, K., Hwang, S. U., Jeon, H. M., Hyun, S. H., and Kim, H. (2016). Comparative analysis of human, mouse, and pig glial fibrillary acidic protein gene structures. Anim. Biotechnol. 27, 126-132. doi: 10.1080/10495398.2015.1126719

Evans, R. J., Wyllie, F. S., Wynford-Thomas, D., Kipling, D., and Jones, C. J. (2003). A P53-dependent, telomere-independent proliferative life span barrier in human astrocytes consistent with the molecular genetics of glioma development. Cancer Res. 63, 4854-4861.

Ferris, H. A., Perry, R. J., Moreira, G. V., Shulman, G. I., Horton, J. D., and Kahn, C. R. (2017). Loss of astrocyte cholesterol synthesis disrupts neuronal function and alters whole-body metabolism. Proc. Natl. Acad. Sci. U.S.A. 114, 1189-1194. doi: 10.1073/pnas.1620506114

Fivenson, E. M., Lautrup, S., Sun, N., Scheibye-Knudsen, M., Stevnsner, T., Nilsen, H., et al. (2017). Mitophagy in neurodegeneration and aging. Neurochem. Int. 109, 202-209. doi: 10.1016/j.neuint.2017.02.007

Flanary, B. (2005). The role of microglial cellular senescence in the aging and Alzheimer diseased brain. Rejuvenation Res. 8, 82-85. doi: 10.1089/rej. 2005.8.82

Freund, A., Laberge, R. M., Demaria, M., and Campisi, J. (2012). Lamin B1 loss is a senescence-associated biomarker. Mol. Biol. Cell 23, 2066-2075. doi: 10.1091/ mbc.E11-10-0884

Frost, G. R., and Li, Y. M. (2017). The role of astrocytes in amyloid production and Alzheimer's disease. Open Biol. 7:170228. doi: 10.1098/rsob.170228

Garwood, C. J., Ratcliffe, L. E., Simpson, J. E., Heath, P. R., Ince, P. G., and Wharton, S. B. (2017). Review: astrocytes in Alzheimer's disease and other ageassociated dementias: a supporting player with a central role. Neuropathol. Appl. Neurobiol. 43, 281-298. doi: 10.1111/nan.12338

Gezen-Ak, D., Dursun, E., Hanagasi, H., Bilgic, B., Lohman, E., Araz, O. S., et al. (2013). BDNF, TNFalpha, HSP90, CFH, and IL-10 serum levels in patients with early or late onset Alzheimer's disease or mild cognitive impairment. J. Alzheimers Dis. 37, 185-195. doi: 10.3233/JAD-130497

Giudetti, A. M., Romano, A., Lavecchia, A. M., and Gaetani, S. (2016). The role of brain cholesterol and its oxidized products in Alzheimer's Disease. Curr. Alzheimer Res. 13, 198-205. doi: 10.2174/1567205012666150921103426

Glenner, G. G., and Wong, C. W. (1984). Alzheimer's disease: initial report of the purification and characterization of a novel cerebrovascular amyloid protein. Biochem. Biophys. Res. Commun. 120, 885-890. doi: 10.1016/s0006-291x(84) 80190-4

Goedert, M., Klug, A., and Crowther, R. A. (2006). Tau protein, the paired helical filament and Alzheimer's disease. J. Alzheimers Dis. 9, 195-207.

Gonzalez-Reyes, R. E., Nava-Mesa, M. O., Vargas-Sanchez, K., Ariza-Salamanca, D., and Mora-Munoz, L. (2017). Involvement of astrocytes in Alzheimer's Disease from a neuroinflammatory and oxidative stress perspective. Front. Mol. Neurosci. 10:427. doi: 10.3389/fnmol.2017.00427

Gorg, B., Karababa, A., and Haussinger, D. (2018). Hepatic encephalopathy and astrocyte senescence. J. Clin. Exp. Hepatol. 8, 294-300. doi: 10.1016/j.jceh.2018. 05.003 
Gorg, B., Karababa, A., Shafigullina, A., Bidmon, H. J., and Haussinger, D. (2015). Ammonia-induced senescence in cultured rat astrocytes and in human cerebral cortex in hepatic encephalopathy. Glia 63, 37-50. doi: 10.1002/glia.22731

Gorgoulis, V., Adams, P. D., Alimonti, A., Bennett, D. C., Bischof, O., Bishop, C., et al. (2019). Cellular senescence: defining a path forward. Cell 179, 813-827. doi: 10.1016/j.cell.2019.10.005

Grolla, A. A., Fakhfouri, G., Balzaretti, G., Marcello, E., Gardoni, F., Canonico, P. L., et al. (2013). Abeta leads to $\mathrm{Ca}(2)(+)$ signaling alterations and transcriptional changes in glial cells. Neurobiol. Aging 34, 511-522. doi: 10. 1016/j.neurobiolaging.2012.05.005

Hampel, H., Mesulam, M. M., Cuello, A. C., Farlow, M. R., Giacobini, E., Grossberg, G. T., et al. (2018). The cholinergic system in the pathophysiology and treatment of Alzheimer's disease. Brain 141, 1917-1933.

Han, X., Tai, H., Wang, X., Wang, Z., Zhou, J., Wei, X., et al. (2016). AMPK activation protects cells from oxidative stress-induced senescence via autophagic flux restoration and intracellular $\mathrm{NAD}(+)$ elevation. Aging Cell 15, 416-427. doi: 10.1111/acel.12446

Hansen, L., Salmon, D., Galasko, D., Masliah, E., Katzman, R., DeTeresa, R., et al. (1990). The Lewy body variant of Alzheimer's disease: a clinical and pathologic entity. Neurology 40, 1-8.

Hara, Y., McKeehan, N., and Fillit, H. M. (2019). Translating the biology of aging into novel therapeutics for Alzheimer disease. Neurology 92, 84-93. doi: 10. 1212/WNL.0000000000006745

Hardy, J. A., and Higgins, G. A. (1992). Alzheimer's disease: the amyloid cascade hypothesis. Science 256, 184-185.

He, N., Jin, W. L., Lok, K. H., Wang, Y., Yin, M., and Wang, Z. J. (2013). Amyloid-beta(1-42) oligomer accelerates senescence in adult hippocampal neural stem/progenitor cells via formylpeptide receptor 2. Cell Death Dis. 4:e924. doi: 10.1038/cddis.2013.437

Heneka, M. T., Carson, M. J., Khoury, J. E., Landreth, G. E., Brosseron, F., Feinstein, D. L., et al. (2015). Neuroinflammation in Alzheimer's disease. Lancet Neurol. 14, 388-405.

Hernandez-Segura, A., Nehme, J., and Demaria, M. (2018). Hallmarks of cellular senescence. Trends Cell Biol. 28, 436-453. doi: 10.1016/j.tcb.2018.02.001

Herranz, N., Gallage, S., Mellone, M., Wuestefeld, T., Klotz, S., Hanley, C. J., et al. (2015). mTOR regulates MAPKAPK2 translation to control the senescenceassociated secretory phenotype. Nat. Cell Biol. 17, 1205-1217. doi: 10.1038/ ncb3225

Hoare, M., Ito, Y., Kang, T. W., Weekes, M. P., Matheson, N. J., Patten, D. A., et al. (2016). NOTCH1 mediates a switch between two distinct secretomes during senescence. Nat. Cell Biol. 18, 979-992. doi: 10.1038/ncb3397

Hong, S., Beja-Glasser, V. F., Nfonoyim, B. M., Frouin, A., Li, S., Ramakrishnan, S., et al. (2016). Complement and microglia mediate early synapse loss in Alzheimer mouse models. Science 352, 712-716. doi: 10.1126/science.aad8373

Horng, S., Therattil, A., Moyon, S., Gordon, A., Kim, K., Argaw, A. T., et al. (2017). Astrocytic tight junctions control inflammatory CNS lesion pathogenesis. J. Clin. Invest. 127, 3136-3151. doi: 10.1172/JCI91301

Horstmann, S., Budig, L., Gardner, H., Koziol, J., Deuschle, M., Schilling, C., et al. (2010). Matrix metalloproteinases in peripheral blood and cerebrospinal fluid in patients with Alzheimer's disease. Int. Psychogeriatr. 22, 966-972. doi: 10.1017/S1041610210000827

Hou, J., Cui, C., Kim, S., Sung, C., and Choi, C. (2018). Ginsenoside F1 suppresses astrocytic senescence-associated secretory phenotype. Chem. Biol. Interact. 283, 75-83. doi: 10.1016/j.cbi.2018.02.002

Hou, J., Kim, S., Sung, C., and Choi, C. (2017). Ginsenoside Rg3 prevents oxidative stress-induced astrocytic senescence and ameliorates senescence paracrine effects on glioblastoma. Molecules 22:E1516. doi: 10.3390/molecules22091516

Hsiao, K., Chapman, P., Nilsen, S., Eckman, C., Harigaya, Y., Younkin, S., et al. (1996). Correlative memory deficits, Abeta elevation, and amyloid plaques in transgenic mice. Science 274, 99-102.

Hussaini, S. M. Q., and Jang, M. H. (2018). New roles for old glue: astrocyte function in synaptic plasticity and neurological disorders. Int. Neurourol. J. 22, S106-S114. doi: 10.5213/inj.1836214.107

Iram, T., Trudler, D., Kain, D., Kanner, S., Galron, R., Vassar, R., et al. (2016). Astrocytes from old Alzheimer's disease mice are impaired in abeta uptake and in neuroprotection. Neurobiol. Dis. 96, 84-94. doi: 10.1016/j.nbd.2016.08.001

Jeon, O. H., Kim, C., Laberge, R. M., Demaria, M., Rathod, S., Vasserot, A. P., et al. (2017). Local clearance of senescent cells attenuates the development of post-traumatic osteoarthritis and creates a pro-regenerative environment. Nat. Med. 23, 775-781. doi: 10.1038/nm.4324

Jurk, D., Wang, C., Miwa, S., Maddick, M., Korolchuk, V., Tsolou, A., et al. (2012). Postmitotic neurons develop a p21-dependent senescence-like phenotype driven by a DNA damage response. Aging Cell 11, 996-1004. doi: 10.1111/j. 1474-9726.2012.00870.x

Kawano, H., Katsurabayashi, S., Kakazu, Y., Yamashita, Y., Kubo, N., Kubo, M., et al. (2012). Long-term culture of astrocytes attenuates the readily releasable pool of synaptic vesicles. PLoS One 7:e48034. doi: 10.1371/journal.pone. 0048034

Kim, J. H., Jittiwat, J., Ong, W. Y., Farooqui, A. A., and Jenner, A. M. (2010). Changes in cholesterol biosynthetic and transport pathways after excitotoxicity. J. Neurochem. 112, 34-41. doi: 10.1111/j.1471-4159.2009.06449.x

Kim, Y. M., Youn, S. W., Sudhahar, V., Das, A., Chandhri, R., Cuervo Grajal, H., et al. (2018). Redox regulation of mitochondrial fission protein drp1 by protein disulfide isomerase limits endothelial senescence. Cell Rep. 23, 3565-3578. doi: 10.1016/j.celrep.2018.05.054

Knorpp, T., Robinson, S. R., Crack, P. J., and Dringen, R. (2006). Glutathione peroxidase- 1 contributes to the protection of glutamine synthetase in astrocytes during oxidative stress. J. Neural. Transm. 113, 1145-1155. doi: 10.1007/ s00702-005-0389-y

Koffie, R. M., Meyer-Luehmann, M., Hashimoto, T., Adams, K. W., Mielke, M. L., Garcia-Alloza, M., et al. (2009). Oligomeric amyloid beta associates with postsynaptic densities and correlates with excitatory synapse loss near senile plaques. Proc. Natl .Acad. Sci. U.S.A. 106, 4012-4017. doi: 10.1073/pnas. 0811698106

Kosar, M., Bartkova, J., Hubackova, S., Hodny, Z., Lukas, J., and Bartek, J. (2011). Senescence-associated heterochromatin foci are dispensable for cellular senescence, occur in a cell type- and insult-dependent manner and follow expression of p16(ink4a). Cell Cycle 10, 457-468. doi: 10.4161/cc.10.3.14707

Kritsilis, M., Rizou, V. S., Koutsoudaki, P. N., Evangelou, K., Gorgoulis, V. G., Papadopoulos, D., et al. (2018). Ageing, cellular senescence and neurodegenerative disease. Int. J. Mol. Sci. 19:E2937. doi: 10.3390/ ijms19102937

Kurz, D. J., Decary, S., Hong, Y., and Erusalimsky, J. D. (2000). Senescenceassociated (beta)-galactosidase reflects an increase in lysosomal mass during replicative ageing of human endothelial cells. J. Cell Sci. 113(Pt 20), 3613-3622.

Lalo, U., Rasooli-Nejad, S., and Pankratov, Y. (2014). Exocytosis of gliotransmitters from cortical astrocytes: implications for synaptic plasticity and aging. Biochem. Soc. Trans. 42, 1275-1281. doi: 10.1042/BST20140163

Larsson, A., Wilhelmsson, U., Pekna, M., and Pekny, M. (2004). Increased cell proliferation and neurogenesis in the hippocampal dentate gyrus of old GFAP(/-)Vim(-/-) mice. Neurochem. Res. 29, 2069-2073. doi: 10.1007/s11064-0046880-2

Leake, A., Morris, C. M., and Whateley, J. (2000). Brain matrix metalloproteinase 1 levels are elevated in Alzheimer's disease. Neurosci. Lett. 291, 201-203. doi: 10.1016/s0304-3940(00)01418-x

Lee, B. Y., Han, J. A., Im, J. S., Morrone, A., Johung, K., Goodwin, E. C., et al. (2006). Senescence-associated beta-galactosidase is lysosomal beta-galactosidase. Aging Cell 5, 187-195.

Li, T., and Chen, Z. J. (2018). The cGAS-cGAMP-STING pathway connects DNA damage to inflammation, senescence, and cancer. J. Exp. Med. 215, 1287-1299. doi: 10.1084/jem.20180139

Liddelow, S. A., Guttenplan, K. A., Clarke, L. E., Bennett, F. C., Bohlen, C. J., Schirmer, L., et al. (2017). Neurotoxic reactive astrocytes are induced by activated microglia. Nature 541, 481-487. doi: 10.1038/nature21029

Liu, C. C., Hu, J., Zhao, N., Wang, J., Wang, N., Cirrito, J. R., et al. (2017). Astrocytic LRP1 mediates brain abeta clearance and impacts amyloid deposition. J. Neurosci. 37, 4023-4031. doi: 10.1523/JNEUROSCI.3442-16.2017

Lok, K., Zhao, H., Zhang, C., He, N., Shen, H., Wang, Z., et al. (2013). Effects of accelerated senescence on learning and memory, locomotion and anxiety-like behavior in APP/PS1 mouse model of Alzheimer's disease. J. Neurol. Sci. 335, 145-154. doi: 10.1016/j.jns.2013.09.018

Long, J. M., and Holtzman, D. M. (2019). Alzheimer Disease: an update on pathobiology and treatment strategies. Cell 179, 312-339. doi: 10.1016/j.cell. 2019.09.001

Loo, T. M., Miyata, K., Tanaka, Y., and Takahashi, A. (2019). Cellular senescence and senescence-associated secretory phenotype via the cGAS-STING 
signaling pathway in cancer. Cancer Sci. 111, 304-311. doi: 10.1111/cas. 14266

Lye, J. J., Latorre, E., Lee, B. P., Bandinelli, S., Holley, J. E., Gutowski, N. J., et al. (2019). Astrocyte senescence may drive alterations in GFAPalpha, CDKN2A p14(ARF), and TAU3 transcript expression and contribute to cognitive decline. Geroscience 41, 561-573. doi: 10.1007/s11357-019-00100-3

Matias, I., Morgado, J., and Gomes, F. C. A. (2019). Astrocyte heterogeneity: impact to brain aging and disease. Front. Aging Neurosci 11:59. doi: 10.3389/fnagi.2019. 00059

McGeer, P. L., and McGeer, E. G. (2007). NSAIDs and Alzheimer disease: epidemiological, animal model and clinical studies. Neurobiol. Aging 28, 639647. doi: 10.1016/j.neurobiolaging.2006.03.013

Mendelsohn, A. R., and Larrick, J. W. (2018). Cellular senescence as the key intermediate in tau-mediated neurodegeneration. Rejuvenation Res. 21, 572579. doi: 10.1089/rej.2018.2155

Miller, E. C., Teravskis, P. J., Dummer, B. W., Zhao, X., Huganir, R. L., and Liao, D. (2014). Tau phosphorylation and tau mislocalization mediate soluble abeta oligomer-induced AMPA glutamate receptor signaling deficits. Eur. J. Neurosci. 39, 1214-1224. doi: 10.1111/ejn.12507

Mogi, M., Harada, M., Kondo, T., Riederer, P., Inagaki, H., Minami, M., et al. (1994). Interleukin-1 $\beta$, interleukin-6, epidermal growth factor and transforming growth factor- $\alpha$ are elevated in the brain from parkinsonian patients. Neurosci. Lett. 180, 147-150. doi: 10.1016/0304-3940(94)90508-8

Mombach, J. C., Vendrusculo, B., and Bugs, C. A. (2015). A model for p38MAPKinduced astrocyte senescence. PLoS One 10:e0125217. doi: 10.1371/journal. pone. 0125217

Montagne, A., Barnes, S. R., Sweeney, M. D., Halliday, M. R., Sagare, A. P., Zhao, Z., et al. (2015). Blood-brain barrier breakdown in the aging human hippocampus. Neuron 85, 296-302. doi: 10.1016/j.neuron.2014.12.032

Morel, L., Chiang, M. S. R., Higashimori, H., Shoneye, T., Iyer, L. K., Yelick, J., et al. (2017). Molecular and functional properties of regional astrocytes in the adult brain. J. Neurosci. 37, 8706-8717. doi: 10.1523/JNEUROSCI.395616.2017

Munger, E. L., Edler, M. K., Hopkins, W. D., Ely, J. J., Erwin, J. M., Perl, D. P., et al. (2019). Astrocytic changes with aging and Alzheimer's disease-type pathology in chimpanzees. J. Comp. Neurol. 527, 1179-1195. doi: 10.1002/cne. 24610

Musi, N., Valentine, J. M., Sickora, K. R., Baeuerle, E., Thompson, C. S., Shen, Q., et al. (2018). Tau protein aggregation is associated with cellular senescence in the brain. Aging Cell 17, e12840. doi: 10.1111/acel.12840

Myung, N. H., Zhu, X., Kruman, I. I., Castellani, R. J., Petersen, R. B., Siedlak, S. L., et al. (2008). Evidence of DNA damage in Alzheimer disease: phosphorylation of histone H2AX in astrocytes. Age 30, 209-215. doi: 10.1007/s11357-008-9050-7

Nichols, N. R., Day, J. R., Laping, N. J., Johnson, S. A., and Finch, C. E. (1993). GFAP mRNA increases with age in rat and human brain. Neurobiol. Aging 14, 421-429. doi: 10.1016/0197-4580(93)90100-p

Nicoll, J. A., and Weller, R. O. (2003). A new role for astrocytes: beta-amyloid homeostasis and degradation. Trends Mol. Med. 9, 281-282. doi: 10.1016/ s1471-4914(03)00109-6

Pajarillo, E., Rizor, A., Lee, J., Aschner, M., and Lee, E. (2019). The role of astrocytic glutamate transporters GLT-1 and GLAST in neurological disorders: potential targets for neurotherapeutics. Neuropharmacology 161:107559. doi: 10.1016/j. neuropharm.2019.03.002

Palmer, A. L., and Ousman, S. S. (2018). Astrocytes and Aging. Front. Aging Neurosci. 10:337. doi: 10.3389/fnagi.2018.00337

Passos, J. F., Saretzki, G., Ahmed, S., Nelson, G., Richter, T., Peters, H., et al. (2007). Mitochondrial dysfunction accounts for the stochastic heterogeneity in telomere-dependent senescence. PLoS Biol. 5:e110. doi: 10.1371/journal.pbio. 0050110

Perez-Nievas, B. G., and Serrano-Pozo, A. (2018). Deciphering the astrocyte reaction in Alzheimer's Disease. Front. Aging Neurosci. 10:114. doi: 10.3389/ fnagi.2018.00114

Pertusa, M., Garcia-Matas, S., Rodriguez-Farre, E., Sanfeliu, C., and Cristofol, R. (2007). Astrocytes aged in vitro show a decreased neuroprotective capacity. J. Neurochem. 101, 794-805. doi: 10.1111/j.1471-4159.2006.04369.x

Piacentini, R., Li Puma, D. D., Mainardi, M., Lazzarino, G., Tavazzi, B., Arancio, O., et al. (2017). Reduced gliotransmitter release from astrocytes mediates tau-induced synaptic dysfunction in cultured hippocampal neurons. Glia 65, 1302-1316. doi: 10.1002/glia.23163
Ponten, J., and Macintyre, E. H. (1968). Long term culture of normal and neoplastic human glia. Acta Pathol. Microbiol. Scand. 74, 465-486. doi: 10.1111/j.16990463.1968.tb03502.x

Porchet, R., Probst, A., Bouras, C., Draberova, E., Draber, P., and Riederer, B. M. (2003). Analysis of glial acidic fibrillary protein in the human entorhinal cortex during aging and in Alzheimer's disease. Proteomics 3, 1476-1485. doi: 10.1002/ pmic. 200300456

Qiu, J., Xu, J., Zheng, Y., Wei, Y., Zhu, X., Lo, E. H., et al. (2010). Highmobility group box 1 promotes metalloproteinase-9 upregulation through Toll-like receptor 4 after cerebral ischemia. Stroke 41, 2077-2082. doi: 10.1161/ STROKEAHA.110.590463

Rajmohan, R., and Reddy, P. H. (2017). Amyloid-beta and phosphorylated tau accumulations cause abnormalities at synapses of Alzheimer's disease Neurons. J. Alzheimers Dis 57, 975-999. doi: 10.3233/JAD- 160612

Ries, M., and Sastre, M. (2016). Mechanisms of abeta clearance and degradation by glial cells. Front. Aging Neurosci. 8:160. doi: 10.3389/fnagi.2016.00160

Rochfort, K. D., and Cummins, P. M. (2015). The blood-brain barrier endothelium: a target for pro-inflammatory cytokines. Biochem. Soc. Trans. 43, 702-706. doi: 10.1042/BST20140319

Salminen, A., Ojala, J., Kaarniranta, K., Haapasalo, A., Hiltunen, M., and Soininen, H. (2011). Astrocytes in the aging brain express characteristics of senescenceassociated secretory phenotype. Eur. .J Neurosci. 34, 3-11. doi: 10.1111/j.14609568.2011.07738.x

Scott, K. M., and Williams-Gray, C. H. (2018). Targeting aged astrocytes may be a new therapeutic strategy in Parkinson's Disease. Mov. Disord. 33, 758-759. doi: $10.1002 / \mathrm{mds} .27387$

Seoane, M., Costoya, J. A., and Arce, V. M. (2017). Uncoupling oncogene-induced senescence (OIS) and DNA damage response (DDR) triggered by DNA hyperreplication: lessons from primary mouse embryo astrocytes (MEA). Sci. Rep. 7:12991. doi: 10.1038/s41598-017-13408-x

Shi, X., Wang, B., Liu, Y., Zhang, J., Huang, Y., Cao, P., et al. (2017). Carnosine modulates glutamine synthetase expression in senescent astrocytes exposed to oxygen-glucose deprivation/recovery. Brain Res. Bull. 130, 138-145. doi: 10.1016/j.brainresbull.2017.01.014

Sofroniew, M. V., and Vinters, H. V. (2010). Astrocytes: biology and pathology. Acta Neuropathol. 119, 7-35. doi: 10.1007/s00401-009-0619-8

Souza, D. G., Bellaver, B., Raupp, G. S., Souza, D. O., and Quincozes-Santos, A. (2015). Astrocytes from adult Wistar rats aged in vitro show changes in glial functions. Neurochem. Int. 90, 93-97. doi: 10.1016/j.neuint.2015.07.016

Spampinato, S. F., Merlo, S., Sano, Y., Kanda, T., and Sortino, M. A. (2017). Astrocytes contribute to Abeta-induced blood-brain barrier damage through activation of endothelial MMP9. J. Neurochem. 142, 464-477. doi: 10.1111/jnc. 14068

Stamouli, E. C., and Politis, A. M. (2016). [Pro-inflammatory cytokines in Alzheimer's disease]. Psychiatriki 27, 264-275.

Tai, H., Wang, Z., Gong, H., Han, X., Zhou, J., Wang, X., et al. (2017). Autophagy impairment with lysosomal and mitochondrial dysfunction is an important characteristic of oxidative stress-induced senescence. Autophagy 13, 99-113. doi: 10.1080/15548627.2016.1247143

Taylor, J. M., Moore, Z., Minter, M. R., and Crack, P. J. (2018). Type-I interferon pathway in neuroinflammation and neurodegeneration: focus on Alzheimer's disease. J. Neural. Transm. 125, 797-807. doi: 10.1007/s00702-017-1745-4

Terry, R. D., Masliah, E., Salmon, D. P., Butters, N., DeTeresa, R., Hill, R., et al. (1991). Physical basis of cognitive alterations in Alzheimer's disease: synapse loss is the major correlate of cognitive impairment. Ann. Neurol. 30, 572-580. doi: 10.1002/ana.410300410

Trias, E., Beilby, P. R., Kovacs, M., Ibarburu, S., Varela, V., Barreto-Nunez, R., et al. (2019). Emergence of microglia bearing senescence markers during paralysis progression in a rat model of inherited ALS. Front. Aging Neurosci. 11:42. doi: 10.3389/fnagi.2019.00042

Turnquist, C., Beck, J. A., Horikawa, I., Obiorah, I. E., Von Muhlinen, N., Vojtesek, B., et al. (2019). Radiation-induced astrocyte senescence is rescued by Delta133p53. Neuro Oncol. 21, 474-485. doi: 10.1093/neuonc/noz001

Turnquist, C., Horikawa, I., Foran, E., Major, E. O., Vojtesek, B., Lane, D. P., et al. (2016). p53 isoforms regulate astrocyte-mediated neuroprotection and neurodegeneration. Cell Death Differ. 23, 1515-1528. doi: 10.1038/cdd.2016.37

Vasileiou, P. V. S., Evangelou, K., Vlasis, K., Fildisis, G., Panayiotidis, M. I., Chronopoulos, E., et al. (2019). Mitochondrial homeostasis and cellular senescence. Cells 8:686. doi: 10.3390/cells8070686 
Vazquez-Villasenor, I., Garwood, C. J., Heath, P. R., Simpson, J. E., Ince, P. G., and Wharton, S. B. (2019). Expression of p16 and p21 in the frontal association cortex of ALS/MND brains suggests neuronal cell cycle dysregulation and astrocyte senescence in early stages of the disease. Neuropathol. Appl. Neurobiol. 46, 171-185. doi: 10.1111/nan.12559

Verkhratsky, A., and Kirchhoff, F. (2007). Glutamate-mediated neuronal-glial transmission. J. Anat. 210, 651-660. doi: 10.1111/j.1469-7580.2007.00734.x

Verkhratsky, A., and Nedergaard, M. (2018). Physiology of astroglia. Physiol. Rev. 98, 239-389. doi: 10.1152/physrev.00042.2016

Wood, J. A., Wood, P. L., Ryan, R., Graff-Radford, N. R., Pilapil, C., Robitaille, Y., et al. (1993). Cytokine indices in Alzheimer's temporal cortex: no changes in mature IL-1 $\beta$ or IL-1RA but increases in the associated acute phase proteins IL-6, $\alpha 2$-macroglobulin and C-reactive protein. Brain Res. 629, 245-252. doi: 10.1016/0006-8993(93)91327-o

Yamazaki, Y., Baker, D. J., Tachibana, M., Liu, C. C., van Deursen, J. M., Brott, T. G., et al. (2016). Vascular cell senescence contributes to blood-brain barrier breakdown. Stroke 47, 1068-1077. doi: 10.1161/STROKEAHA.115.01 0835

Yamazaki, Y., and Kanekiyo, T. (2017). Blood-brain barrier dysfunction and the pathogenesis of Alzheimer's Disease. Int. J. Mol. Sci. 18:E1965.

Yoon, K. B., Park, K. R., Kim, S. Y., and Han, S. Y. (2016). Induction of nuclear enlargement and senescence by sirtuin inhibitors in glioblastoma Cells. Immune Netw. 16, 183-188. doi: 10.4110/in.2016.16.3.183

Yu, C., Narasipura, S. D., Richards, M. H., Hu, X. T., Yamamoto, B., and AlHarthi, L. (2017). HIV and drug abuse mediate astrocyte senescence in a beta-catenin-dependent manner leading to neuronal toxicity. Aging Cell 16, 956-965. doi: 10.1111/acel.12593

Zhang, P., Kishimoto, Y., Grammatikakis, I., Gottimukkala, K., Cutler, R. G., Zhang, S., et al. (2019). Senolytic therapy alleviates Abeta-associated oligodendrocyte progenitor cell senescence and cognitive deficits in an Alzheimer's disease model. Nat. Neurosci. 22, 719-728. doi: 10.1038/s41593019-0372-9

Zhang, X., Lao, K., Qiu, Z., Rahman, M. S., Zhang, Y., and Gou, X. (2019). Potential astrocytic receptors and transporters in the pathogenesis of Alzheimer's Disease. J. Alzheimers Dis. 67, 1109-1122. doi: 10.3233/jad-181084

Zhu, Y., Armstrong, J. L., Tchkonia, T., and Kirkland, J. L. (2014). Cellular senescence and the senescent secretory phenotype in age-related chronic diseases. Curr. Opin. Clin. Nutr. Metab. Care 17, 324-328. doi: 10.1097/MCO. 0000000000000065

Conflict of Interest: The authors declare that the research was conducted in the absence of any commercial or financial relationships that could be construed as a potential conflict of interest.

Copyright (C) 2020 Han, Zhang, Liu, Mi and Gou. This is an open-access article distributed under the terms of the Creative Commons Attribution License (CC BY). The use, distribution or reproduction in other forums is permitted, provided the original author(s) and the copyright owner(s) are credited and that the original publication in this journal is cited, in accordance with accepted academic practice. No use, distribution or reproduction is permitted which does not comply with these terms. 